\title{
THE ABSOLUTE CONVERGENCE OF CERTAIN LACUNARY FOURIER SERIES
}

\author{
George Benke
}

Let $\mathrm{G}$ be a compact abelian group, and let $\Gamma$ be its dual group. Suppose $\mathrm{E} \subset \Gamma$ and $f$ is a function on $G$. The function $f$ is called an E-function if $\hat{f}(\gamma)=0$ for all $\gamma \notin \mathrm{E}$ ( $\hat{\mathrm{f}}$ is the Fourier transform of $\mathrm{f}$ ). By $\mathrm{A}(\mathrm{G})$ we denote the space of functions whose transforms belong to $\ell^{l}(\Gamma)$, and $\|\mathrm{f}\|_{A(G)}$ is defined to be $\|\hat{f}\|_{\ell^{1}(\Gamma)}$. For each set $S(G)$ of functions defined on $G$, we denote by $S_{E}(G)$ the $E$-functions in $S(G)$. A set $E \subset \Gamma$ is a Sidon set if $A_{E}(G)=C_{E}(G)$, where $C(G)$ is the space of continuous functions on $G$. For $2<p<\infty$, a set $E \subset \Gamma$ is a $\Lambda(p)-$ set if $L_{E}^{2}(G)=L_{E}^{p}(G)$. A set $E \subset \Gamma$ is a $\Lambda$-set if it is a $\Lambda(p)$-set for all $\mathrm{p}$ and if in addition the inclusions $\mathrm{L}_{\mathrm{E}}^{2}(\mathrm{G}) \rightarrow \mathrm{L}_{\mathrm{E}}^{\mathrm{P}}(\mathrm{G})$ have norm at most $\mathrm{Cp}^{1 / 2}$, where $\mathrm{C}$ depends only on the set $\mathrm{E}$.

It is known that every Sidon set is a $\Lambda$-set [9, p. 128], and that there exist sets that are $\Lambda(\mathrm{p})$-sets for all $\mathrm{p}$ but are not Sidon sets [2, p. 803]. Actually, in the light of results in [1, p. 131], the sets constructed in [2] are not $\Lambda$-sets. It is therefore natural to ask whether there exist $\Lambda$-sets that are not Sidon sets. In general, this is an open question, but in certain torsion groups every $\Lambda$-set is also a Sidon set [6]. That Sidon sets are close to $\Lambda$-sets from a structural standpoint was shown in [1]. In this paper, we show that in an analytical sense they are also close. In particular, we construct a Banach space $B(G)$ of functions on $G$ such that $A(G) \hookrightarrow B(G) \hookrightarrow C(G)$ and such that $E \subset \Gamma$ is a $\Lambda$-set if and only if $A_{E}(G)=B_{E}(G)$. The construction of $B(G)$ is motivated by the work in [3] and [4], and the connection between $B(G)$ and $\Lambda$ sets is analogous to the connection between $A$. Figà-Talamanca's $A^{P}(G)$-spaces and $\Lambda$ (p)-sets [5].

In Section 1 of this paper, we define two spaces $K(G)$ and $R(G)$ of functions on $G$ that, (in the language of $M$. A. Rieffel [7]) are Banach modules. The space $B(G)$ is then defined, and it turns out to be a realization of the Banach module tensor product $K(G) \otimes_{L^{1}(G)} R(G)$. In Section 2, we establish the connection between $B(G)$ and $\Lambda$-sets.

\section{DEFINITIONS AND PROPERTIES OF THE BASIC SPACES}

$$
\begin{aligned}
& \text { For } \mathrm{f} \in \bigcap_{2<\mathrm{p}<\infty} \mathrm{L}^{\mathrm{P}}(\mathrm{G}) \text {, let } \\
& \qquad\|\mathrm{f}\|_{\Lambda}=\sup \left\{\mathrm{p}^{-1 / 2}\|\mathrm{f}\|_{\mathrm{p}} \mid 2<\mathrm{p}<\infty\right\},
\end{aligned}
$$

and let $K(G)$ be the set of all functions $f$ on $G$ for which $\|f\|_{\Lambda}$ is finite. It is easy to verify that \|\|$_{\Lambda}$ is a norm on $\mathrm{K}(\mathrm{G})$ and that, endowed with this norm, $\mathrm{K}(\mathrm{G})$ becomes a two-sided Banach $L^{1}(G)$-module with respect to convolution. Next, we shall define a space $R(G)$ of functions that is also a two-sided Banach $L^{1}(G)$-module

Received June 6, 1973.

Michigan Math. J. 21 (1974). 
and whose dual is $\mathrm{K}(\mathrm{G})$. This is done as follows. Let $\mathrm{R}(\mathrm{G})$ be the closure in $\mathrm{K}(\mathrm{G})^{*}$ of the set of continuous functions on $G$ that define in the natural way elements of $\mathrm{K}(\mathrm{G})^{*}$. We shall see that every continuous function defines an element of $\mathrm{K}(\mathrm{G})^{*}$. In the following proposition, we collect some relevant facts about $K(G)$ and $R(G)$.

PROPOSITION 1. Let $\mathrm{K}(\mathrm{G})$ and $\mathrm{R}(\mathrm{G})$ be defined as above, and denote by \|\|$_{\mathrm{R}}$ the norm dual to \|\|$_{\Lambda}$. The following hold.

(1) $\mathrm{L}^{1}(\mathrm{G}) \supset \mathrm{R}(\mathrm{G}) \supset \mathrm{L}^{\mathrm{q}}(\mathrm{G})$ for all $\mathrm{q}(1<\mathrm{q} \leq 2)$, and

$$
2^{1 / 2}\|\|_{1} \leq\|\|_{R} \leq(q /(q-1))^{1 / 2}\|\|_{q} \text {. }
$$

(2) $\mathrm{L}^{\mathrm{p}}(\mathrm{G}) \supset \mathrm{K}(\mathrm{G}) \supset \mathrm{L}^{\infty}(\mathrm{G})$ for all $\mathrm{p}(2 \leq \mathrm{p}<\infty)$, and

$$
\mathrm{p}^{-1 / 2}\|\|_{\mathrm{p}} \leq\|\|_{\Lambda} \leq 2^{-1 / 2}\|\|_{\infty}
$$

(3) $\left(\mathrm{R}(\mathrm{G})^{*},\|\|_{\mathrm{R}^{*}}\right)=\left(\mathrm{K}(\mathrm{G}),\|\|_{\Lambda}\right)$.

(4) If $\mathrm{f} \in \mathrm{K}(\mathrm{G})$ and $\mathrm{g} \in \mathrm{R}(\mathrm{G})$, then $\mathrm{f} * \mathrm{~g} \in \mathrm{C}(\mathrm{G})$ and $\|\mathrm{f} * \mathrm{~g}\|_{\infty} \leq\|\mathrm{f}\|_{\Lambda}\|\mathrm{g}\|_{\mathrm{R}}$.

Proof. (2) follows immediately from the definition of $\mathrm{K}(\mathrm{G})$. To see that (1) holds, take $g \in \mathrm{L}^{\mathrm{P}}(\mathrm{G})$ and $\mathrm{f} \in \mathrm{K}(\mathrm{G})$. Then, for $\mathrm{p}=\mathrm{q} /(\mathrm{q}-1)$,

$$
\left|\int \mathrm{fg}\right| \leq\|\mathrm{g}\|_{\mathrm{q}}\|\mathrm{f}\|_{\mathrm{p}} \leq\left(\mathrm{p}^{1 / 2}\|\mathrm{~g}\|_{\mathrm{q}}\right)\|\mathrm{f}\|_{\Lambda} .
$$

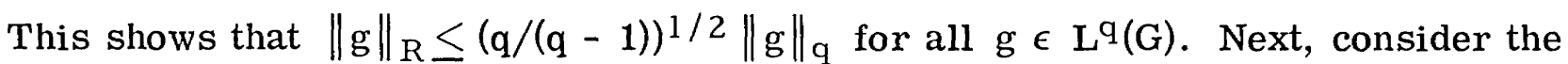
inclusion map $\mathrm{i}: \mathrm{C}(\mathrm{G}) \rightarrow \mathrm{K}(\mathrm{G})$ and its adjoint $\mathrm{i}^{*}: \mathrm{K}(\mathrm{G})^{*} \rightarrow \mathrm{M}(\mathrm{G})$. We have the relations

$$
\left\|\mathrm{i}^{*}\right\|=\|\mathrm{i}\|=\sup \left\{\|\mathrm{f}\|_{\Lambda} /\|\mathrm{f}\|_{\infty} \mid \mathrm{f} \in \mathrm{C}(\mathrm{G})\right\}
$$

and by (2) the last member is at most $2^{-1 / 2}$. Taking $g \in C(G)$ and noting that $\mathrm{i}^{*}(\mathrm{~g})=\mathrm{g}$, we see that $\|\mathrm{g}\|_{1} \leq 2^{-1 / 2}\|\mathrm{~g}\|_{\mathrm{R}}$. Once we have shown that $\mathrm{L}^{\mathrm{l}}(\mathrm{G}) \supset \mathrm{R}(\mathrm{G})$, this norm inequality will hold for all $g \in R(G)$. We now show that $L^{l}(G) \supset R(G)$. Suppose $\left\{g_{n}\right\}$ is a Cauchy sequence in \|\|$_{R}$ of continuous functions. Since \|\|$_{R}$ is stronger than \|\|$_{1}$, the sequence $\left\{g_{n}\right\}$ converges to some $g$ in $L^{l}(G)$, and some subsequence $\left\{\mathrm{g}_{\mathrm{n}_{\mathrm{k}}}\right\}$ converges pointwise almost everywhere to $\mathrm{g}$. Given $\varepsilon>0$, take $N$ so that $n, m>N$ implies $\left\|g_{n}-g_{m}\right\|_{R}<\varepsilon$. Consider any $f \epsilon K(G)$. Clearly,

$$
\begin{aligned}
\left|\int\left(\mathrm{g}-\mathrm{g}_{\mathrm{n}}\right) \mathrm{f}\right| /\|\mathrm{f}\|_{\Lambda} & \leq \int_{\mathrm{k} \rightarrow \infty} \lim _{\mathrm{n}_{\mathrm{k}}}-\mathrm{g}_{\mathrm{n}}|| \mathrm{f} \mid /\|\mathrm{f}\|_{\Lambda} \\
& \leq \underset{\mathrm{k} \rightarrow \infty}{\liminf } \int\left|\mathrm{g}_{\mathrm{n}_{\mathrm{k}}}-\mathrm{g}_{\mathrm{n}}\right||\mathrm{f}| /\|\mathrm{f}\|_{\Lambda} \leq \liminf \left\|\mathrm{g}_{\mathrm{n}_{\mathrm{k}}}-\mathrm{g}_{\mathrm{n}}\right\|_{\mathrm{R}} \leq \varepsilon
\end{aligned}
$$

Therefore $\mathrm{g}-\mathrm{g}_{\mathrm{n}} \in \mathrm{R}(\mathrm{G})$ and $\left\|\mathrm{g}-\mathrm{g}_{\mathrm{n}}\right\|_{\mathrm{R}} \leq \varepsilon$. It follows that $\mathrm{g} \in \mathrm{R}(\mathrm{G})$ and $\mathrm{L}^{1}(\mathrm{G}) \supset \mathrm{R}(\mathrm{G})$.

Next, we show that $R(G)^{*}=K(G)$. Since the inclusion map $i: L^{q}(G) \rightarrow R(G)$ is dense for all $q>1$, the adjoint $i^{*}: R(G)^{*} \rightarrow L^{P}(G)$ is one-to-one for all $p<\infty$. Therefore $\mathrm{R}(\mathrm{G})^{*} \subset \bigcap_{\mathrm{p}<\infty} \mathrm{L}^{\mathrm{P}}(\mathrm{G})$. Take any $\mathrm{f} \in \mathrm{R}(\mathrm{G})^{*}$ and any $\mathrm{p}<\infty$. Then there 
exists a $g \in L^{q}(G)$ such that $\left|\int f g\right|=\|f\|_{p}\|g\|_{q}$. Hence, by (1),

$$
\|\mathrm{f}\|_{\mathrm{R}^{*}} \geq\left|\int \mathrm{fg}\right| /\|\mathrm{g}\|_{\mathrm{R}} \geq\|\mathrm{f}\|_{\mathrm{p}}\|\mathrm{g}\|_{\mathrm{q}} / \mathrm{p}^{1 / 2}\|\mathrm{~g}\|_{\mathrm{q}}
$$

Therefore $\|\mathrm{f}\|_{\mathrm{R}^{*}} \geq\|\mathrm{f}\|_{\Lambda}$, and $\mathrm{R}(\mathrm{G})^{*} \subset \mathrm{K}(\mathrm{G})$. On the other hand, for $\mathrm{f} \in \mathrm{K}(\mathrm{G})$ and $\mathrm{g} \in \mathrm{R}(\mathrm{G})$, we have the inequality $\left|\int \mathrm{fg}\right| \leq\|\mathrm{f}\|_{\Lambda}\|\mathrm{g}\|_{\mathrm{R}}$. Therefore $\|\mathrm{f}\|_{\Lambda} \geq\|\mathrm{f}\|_{\mathrm{R}^{*}}$ and $\mathrm{K}(\mathrm{G}) \subset \mathrm{R}(\mathrm{G})^{*}$.

Finally, we show that (4) holds. For each $g \in R(G)$, the translation map $\tau_{\mathrm{g}}: \mathrm{G} \rightarrow \mathrm{R}(\mathrm{G})$ defined by $\left(\tau_{\mathrm{g}}(\mathrm{x})\right)(\mathrm{y})=\mathrm{g}(\mathrm{y}-\mathrm{x})$ is continuous. This follows from the facts that $\tau_{f}: G \rightarrow C(G)$ is continuous, that $C(G)$ is dense in $R(G)$, and that \|\|$_{\infty}$ is a stronger norm than $\|_{R}$. Now suppose $f \in K(G)$ and $g \in R(G)$. Then (4) follows immediately from the translation invariance of the \|\|$_{\Lambda^{-}}$and \|\|$_{R^{-n o r m s}}$ and the continuity of $\tau_{\mathrm{g}}: \mathrm{G} \rightarrow \mathrm{R}(\mathrm{G})$.

We define $B(G)$ to be the set of functions $f$ on $g$ with a representation

$$
f(x)=\sum_{n=1}^{\infty} \tilde{f}_{n} * g_{n}(x)
$$

where $\mathrm{f}_{\mathrm{n}}(\mathrm{x})=\mathrm{f}_{\mathrm{n}}(-\mathrm{x}), \mathrm{f}_{\mathrm{n}} \in \mathrm{K}(\mathrm{G}), \mathrm{g}_{\mathrm{n}} \in \mathrm{R}(\mathrm{G})$, and $\sum_{\mathrm{n}=1}^{\infty^{\prime}}\left\|\mathrm{f}_{\mathrm{n}}\right\|_{\Lambda}\left\|\mathrm{g}_{\mathrm{n}}\right\|_{\mathrm{R}}<\infty$. In view of (4) of Proposition 1, the series for $f$ converges uniformly. Therefore $B(G)$ is a set of continuous functions. We give $\mathrm{B}(\mathrm{G})$ a norm by putting

$$
\|\mathrm{f}\|_{\mathrm{B}}=\inf \sum_{\mathrm{n}=1}^{\infty}\left\|\mathrm{f}_{\mathrm{n}}\right\|_{\Lambda}\left\|\mathrm{g}_{\mathrm{n}}\right\|_{\mathrm{R}},
$$

where the infimum is taken over all representations of $f$. The features of $B(G)$ that will concern us follow from some general theorems about tensor products of Banach modules (see [7] and [8]), once we have observed that $B(G)$ is in fact such a tensor product. It is easily seen that $\mathrm{K}(\mathrm{G})$ is a (two-sided) Banach $\mathrm{L}^{1}(\mathrm{G})$-module with respect to convolution. That is, for $\phi \in \mathrm{L}^{1}(\mathrm{G})$ and $\mathrm{f} \epsilon \mathrm{K}(\mathrm{G})$, the action of $\phi$ on $\mathrm{f}$ is given by $\phi * \mathrm{f}$. We see that $\mathrm{R}(\mathrm{G})$ is a (two-sided) Banach $\mathrm{L}^{1}(\mathrm{G})$-module, by letting the action of $\phi \in \mathrm{L}^{1}(\mathrm{G})$ on $\mathrm{g} \in \mathrm{R}(\mathrm{G})$ be given by $\tilde{\phi} * \mathrm{~g}$. An almost word-for-word translation of $[8$, p. 76 , Theorem 3.3] in terms of $K(G)$ and $R(G)$ shows that $K(G) \otimes_{L^{l}(G)} R(G)$ is isometrically isomorphic to $B(G)$, the mapping being such that $\mathrm{f} \otimes \mathrm{g}$ corresponds to $\tilde{\mathrm{f}} * \mathrm{~g}$. Thus $\mathrm{B}(\mathrm{G})$ is a Banach space, and

$$
\operatorname{Hom}_{L^{1}(G)}\left(\mathrm{K}(\mathrm{G}), \mathrm{R}(\mathrm{G})^{*}\right) \cong\left(\mathrm{K}(\mathrm{G}) \otimes_{\mathrm{L}^{1}(\mathrm{G})} \mathrm{R}(\mathrm{G})\right)^{*}
$$

The importance of this result lies in the fact that $\operatorname{Hom}_{L^{l}(G)}\left(K(G), R(G)^{*}\right)$ is the space of multipliers from $\mathrm{K}(\mathrm{G})$ to $\mathrm{R}(\mathrm{G})^{*}$, and that $\mathrm{R}(\mathrm{G})^{*}=\mathrm{K}(\mathrm{G})$. Thus, denoting by $M(K)$ the multipliers from $K(G)$ to $K(G)$, we see that

$$
\mathrm{M}(\mathrm{K}) \cong \mathrm{B}(\mathrm{G})^{*} \text {. }
$$


Moreover, this isomorphism is such that for each $\nu \in \mathrm{B}(\mathrm{G})^{*}$ and its corresponding element $\mathrm{T}_{\nu} \in \mathrm{M}(\mathrm{K})$,

$$
\langle\mathrm{g}, \mathrm{T}, \mathrm{f}\rangle=\langle\tilde{\mathrm{f}} * \mathrm{~g}, \nu\rangle
$$

where $\langle$,$\rangle on the left denotes the dual pairing between R(G)$ and $R(G) *=K(G)$, and $\langle$,$\rangle on the right denotes the dual pairing between B(G)$ and $B(G)^{*}$. Another way of expressing this correspondence is to say that, for $\nu \in \mathrm{B}(\mathrm{G})^{*}$, the function on $\Gamma$ associated with $\mathrm{T}_{\nu} \in \mathrm{M}(\mathrm{K})$ is $\hat{\nu}$.

\section{THE CONNECTION WITH $\Lambda$-SETS}

If $\mathrm{E} \subset \Gamma$, then $\mathrm{K}_{E}(\mathrm{G}), \mathrm{R}_{\mathrm{E}}(\mathrm{G})$, and $\mathrm{B}_{\mathrm{E}}(\mathrm{G})$ are all closed subspaces of $\mathrm{K}(\mathrm{G}), \mathrm{R}(\mathrm{G})$, and $\mathrm{B}(\mathrm{G})$, respectively. This is an immediate consequence of the fact that the norms on these spaces are stronger than the $L^{1}(G)$-norm.

PROPOSITION 2. If $\mathrm{E} \subset \Gamma$ is a $\Lambda$-set, then

$$
K_{-E}(G) \otimes_{L^{l}(G)} R_{E}(G) \cong B_{E}(G)
$$

and the \|\|$_{\mathrm{B}^{-n o r m}}$ is equivalent to the \|\|$_{\mathrm{K}_{-}} \otimes \mathrm{R}_{\mathrm{E}}$-norm on $\mathrm{B}_{\mathrm{E}}(\mathrm{G})$, although not isometric to it.

Proof. Clearly, $K_{-E}(G) \otimes_{L^{1}(G)} R_{E}(G) \subset B_{E}(G)$ and \|\|$_{K_{-E}} \otimes R_{E} \geq\|\|_{B}$.

To prove the other inclusion, consider $\chi_{E}$, the characteristic function of $\mathrm{E}$, which defines a multiplier $T$ from $L^{2}(G)$ to $L_{E}^{2}(G)$ by the relation $\widehat{T f}=\chi_{E} \hat{f}$. Noting that $E$ is a $\Lambda$-set if and only if $L_{E}^{2}(G)=K_{E}(G)$, we see, by restricting $T$ to $K(G)$, that $T \in M(K)$ and that the range of $T$ is $K_{E}(G)$. To estimate the norm of $T$, take $f \in K(G)$, and suppose that $C$ is the $\Lambda$-constant of $E$. Then

$$
\|\mathrm{Tf}\|_{\Lambda} \leq \mathrm{C}\|\mathrm{Tf}\|_{2} \leq \mathrm{C}\|\mathrm{f}\|_{2} \leq \mathrm{C} 2^{1 / 2}\|\mathrm{f}\|_{\Lambda}
$$

and therefore $\|\mathrm{T}\|_{\mathrm{M}(\mathrm{K})} \leq 2^{1 / 2} \mathrm{C}$.

Suppose $f \in B_{E}(G)$, where $f=\sum_{n=1}^{\infty} \tilde{f}_{n} * g_{n}$ is a typical representation. Then

$$
f=T(f)=T\left(\sum_{n=1}^{\infty} \tilde{f}_{n} * g_{n}\right)=\sum_{n=1}^{\infty} T\left(\tilde{f}_{n} * g_{n}\right)=\sum_{n=1}^{\infty}\left(T \tilde{f}_{n}\right) * g_{n} .
$$

Note that $\tilde{\mathrm{ff}}_{\mathrm{n}}=\tilde{\mathrm{h}}_{\mathrm{n}}$, where $\mathrm{h}_{\mathrm{n}} \in \mathrm{K}_{-\mathrm{E}}(\mathrm{G})$. In fact, $\hat{\mathrm{h}}_{\mathrm{n}}=\mathrm{X}_{-\mathrm{E}} \hat{\mathrm{f}}_{\mathrm{n}}$. Moreover, $\left\|\mathrm{h}_{\mathrm{n}}\right\|_{\Lambda} \leq 2^{1 / 2} \mathrm{C}\left\|\mathrm{f}_{\mathrm{n}}\right\|_{\Lambda}$

Since $S \in M(K)$, it follows that the adjoint $S^{*}$ belongs to $M\left(K^{*}\right)$. The restriction of $S^{*}$ to $R(G)$ yields a multiplier from $R(G)$ to $R_{E}(G)$. This follows easily from the facts that $R(G)$ is closed in $K(G)^{*}$ and that the continuous functions are dense in $\mathrm{R}(\mathrm{G})$ and are contained in $\mathrm{K}(\mathrm{G})$. Therefore 


$$
f=T^{*}(f)=\sum_{n=1}^{\infty} \tilde{h}_{n} *\left(T^{*} g_{n}\right)
$$

where $\left\|T^{*} g_{n}\right\|_{R} \leq C 2^{1 / 2}\left\|g_{n}\right\|_{R}$. Hence $f \in K_{-E}(G) \otimes_{L^{l}(G)} R_{E}(G)$ and $\|\mathrm{f}\|_{\mathrm{K}_{-} \mathrm{E}} \otimes \underline{\mathrm{R}}_{\mathrm{E}} \leq 2 \mathrm{C}^{2}\|\mathrm{f}\|_{\mathrm{B}}$.

We remark that for each $\mathrm{E} \subset \Gamma$,

$$
A_{E}(G) \cong L_{-E}^{2}(G) \otimes_{L^{1}(G)} L_{E}^{2}(G) \quad \text { and } \quad\|\|_{A}=\|\|{ }_{L_{-E}^{2} \otimes L_{E}^{2}} \cdot
$$

In preparation for the main theorem, we shall prove a probabilistic result, for which we need the following notation. Let $\Omega$ be the interval $[0,1]$, and let $p$ denote Lebesgue measure. Consider the Rademacher functions $\varepsilon_{\mathrm{n}}: \Omega \rightarrow\{-1,1\}$ defined by $\varepsilon_{\mathrm{n}}(\omega)=(-1)^{\mathrm{m}-1}$ if $\omega \in\left[(\mathrm{m}-1) 2^{-\mathrm{n}}, \mathrm{m} 2^{-\mathrm{n}}\right)$ and $\varepsilon_{\mathrm{n}}(1)=-1$, where $\mathrm{n}=0,1,2, \cdots$. For each $f \in \mathrm{L}^{2}(\mathrm{G})$, select an enumeration $\gamma_{0}, \gamma_{1}, \cdots$ of the support of $\hat{\mathrm{f}}$, and define $\mathbf{f}_{\omega} \in \mathrm{L}^{2}(\mathrm{G})$ by $\hat{\mathrm{f}}_{\omega}\left(\gamma_{\mathrm{n}}\right)=\varepsilon_{\mathrm{n}}(\omega) \hat{\mathrm{f}}\left(\gamma_{\mathrm{n}}\right)$.

PROPOSITION 3. If $\mathrm{f} \in \mathrm{L}^{2}(\mathrm{G})$, then the following statements hold.

(a) $\mathrm{p}\left\{\omega \in \Omega \mid \mathrm{f}_{\omega} \in \mathrm{K}(\mathrm{G})\right\}=1$.

(b) For each $\varepsilon>0$, there exists an $\mathrm{N}$ (depending on $\mathrm{f}$ and $\varepsilon$ ) such that $\left\|\mathrm{f}_{\omega}\right\|_{\Lambda} \leq \mathrm{N}\|\mathrm{f}\|_{2}$ for all $\omega$ except possibly a set of measure less than $\varepsilon$.

(c) For each $\mathrm{f} \in \mathrm{L}^{2}(\mathrm{G})$, there exists an $\omega \in \Omega$ such that $\left\|\mathrm{f}_{\omega}\right\|_{\Lambda} \leq 16\|\mathrm{f}\|_{2}$.

Proof. We shall use the following well-known theorem [10, p. 214]. Let $\left\{u_{\mathrm{n}}\right\}$ be a sequence of complex numbers such that $\sum_{\mathrm{n}=0}^{\infty}\left|\mathrm{u}_{\mathrm{n}}\right|^{2}=\mathrm{r}^{2}<\infty$, and let $\mathrm{g}(\omega)=\sum_{\mathrm{n}=0}^{\infty} \mathrm{u}_{\mathrm{n}} \varepsilon_{\mathrm{n}}(\omega)$. Then $\exp \left(\lambda|\mathrm{g}(\cdot)|^{2}\right) \in \mathrm{L}^{\mathrm{I}}(\Omega)$ for every $\lambda>0$, and

$$
\int_{\Omega} \exp \left(\lambda|\mathrm{g}(\omega)|^{2}\right) \mathrm{d} \omega=\sum_{\mathrm{k}=0}^{\infty} \frac{\lambda^{\mathrm{k}}}{\mathrm{k} !} \int_{\Omega}|\mathrm{g}(\omega)|^{2 \mathrm{k}} \mathrm{d} \omega \leq \sum_{\mathrm{k}=0}^{\infty} \frac{\mathrm{k}^{\mathrm{k}}}{\mathrm{k} !}\left(4 \lambda \mathrm{r}^{2}\right)^{\mathrm{k}} \leq \sum_{\mathrm{k}=0}^{\infty}\left(4 \mathrm{e} \lambda \mathrm{r}^{2}\right)^{\mathrm{k}}
$$

Consider the series

$$
\sum_{n=0}^{\infty} \hat{f}\left(\gamma_{n}\right)\left\langle x, \gamma_{n}\right\rangle \varepsilon_{n}(\omega)
$$

Since $f \in L^{2}(G)$, we can apply the theorem to the series (1) with $e \lambda=\left(8 r^{2}\right)^{-1}$, and we obtain the inequality

$$
\sum_{k=0}^{\infty} \frac{\lambda^{k}}{k !} \int_{\Omega}\left|f_{\omega}(x)\right|^{2 k} d \omega \leq 2
$$

Integrating both sides over $\mathrm{G}$ and switching sums and integrals, we see that

$$
\int_{\Omega}\left(\sum_{\mathrm{k}=0}^{\infty} \frac{\lambda^{\mathrm{k}}}{\mathrm{k} !}\left\|\mathrm{f}_{\omega}\right\|_{2 \mathrm{k}}^{2 \mathrm{k}}\right) \mathrm{d} \omega \leq 2
$$


Put $\mathrm{E}_{\mathrm{n}}=\{\omega \mid$ integrand in (2) is at most $2 \mathrm{n}\}$; then clearly $\mathrm{p}\left(\Omega \backslash \mathrm{E}_{\mathrm{n}}\right) \leq 1 / \mathrm{n}$ and $\mathrm{p}\left(\mathrm{E}_{\mathrm{n}}\right) \geq 1-1 / \mathrm{n}$. If $\omega \in \mathrm{E}_{\mathrm{n}}$, then

$$
\frac{\lambda^{\mathrm{k}}}{\mathrm{k} !}\left\|\mathrm{f}_{\omega}\right\|_{2 \mathrm{k}}^{2 \mathrm{k}} \leq 2 \mathrm{n} \quad \text { for } \mathrm{k}=1,2, \cdots .
$$

Using $\mathrm{e} \lambda=\left(8 \mathrm{r}^{2}\right)^{-1}$ and $\mathrm{k} ! \leq(\mathrm{k}+1)^{\mathrm{k}+1} / \mathrm{e}^{\mathrm{k}}$, we see that

$$
(2 \mathrm{k})^{-1 / 2}\left\|\mathrm{f}_{\omega}\right\|_{2 \mathrm{k}} \leq((\mathrm{k}+1) / \mathrm{k})^{1 / 2}(\mathrm{k}+1)^{1 / 2 \mathrm{k}}(2 \mathrm{n})^{1 / 2 \mathrm{k}} 2 \mathrm{r} .
$$

Hence there exists a constant $\mathrm{K}_{\mathrm{n}}$ such that

$$
(2 \mathrm{k})^{-1 / 2}\left\|\mathrm{f}_{\omega}\right\|_{2 \mathrm{k}} \leq \mathrm{K}_{\mathrm{n}}\|\mathrm{f}\|_{2} \quad \text { for all } \mathrm{k}=1,2, \cdots .
$$

Therefore $\|\mathrm{f} \omega\|_{\Lambda} \leq 2^{1 / 2} \mathrm{~K}_{\mathrm{n}}\|\mathrm{f}\|_{2}$ for all $\omega \in \mathrm{E}_{\mathrm{n}}$; this proves (a) and (b). As for (c), set $n=2$ in (3).

PROPOSition 4. $\mathrm{A}(\mathrm{G}) \subset \mathrm{B}(\mathrm{G}) \subset \mathrm{C}(\mathrm{G})$ and $2^{9 / 2}\|\|_{\mathrm{A}} \geq\|\|_{\mathrm{B}} \geq\|\|_{\infty}$.

Proof. Take $\mathrm{f} \in \mathrm{B}(\mathrm{G})$, where $\mathrm{f}=\sum_{\mathrm{n}=1}^{\infty} \tilde{\mathrm{f}}_{\mathrm{n}} * \mathrm{~g}_{\mathrm{n}}$. Then

$$
\|\mathrm{f}\|_{\infty} \leq \sum_{\mathrm{n}=1}^{\infty}\left\|\tilde{\mathrm{f}}_{\mathrm{n}} * \mathrm{~g}_{\mathrm{n}}\right\|_{\infty} \leq \sum_{\mathrm{n}=1}^{\infty}\left\|\mathrm{f}_{\mathrm{n}}\right\|_{\Lambda}\left\|\mathrm{g}_{\mathrm{n}}\right\|_{\mathrm{R}} .
$$

Since this holds for all representations, $\|\mathrm{f}\|_{\infty} \leq\|\mathrm{f}\|_{B}$. That $B(G) \subset C(G)$ was observed earlier.

Now take $h \in A(G)$. Choose $f$ and $g \in L^{2}(G)$ so that $\hat{\tilde{f}} \hat{g}=\hat{h}$ and $|\hat{\tilde{f}}|=|\hat{\mathrm{g}}|=|\hat{\mathrm{h}}|^{1 / 2}$. By Proposition 3, there exists a sign change $\omega \in \Omega$ such that $\tilde{\mathrm{f}}_{\omega} \in \mathrm{K}(\mathrm{G})$ and $\left\|\tilde{\mathrm{f}}_{\omega}\right\|_{\Lambda} \leq 16\|\tilde{\mathrm{f}}\|_{2}$. Note that $\widehat{\hat{\mathrm{f}}}_{\omega} \hat{\mathrm{g}}_{\omega}=\hat{\widetilde{\mathrm{f}}} \hat{\mathrm{g}}$ and hence $\widetilde{\mathrm{f}}_{\omega} * \mathrm{~g}_{\omega}=$ h. Thus $\widetilde{\mathrm{f}}_{\omega} * \mathrm{~g}_{\omega}$ is a representation of $\mathrm{h}$ as an element of $\mathrm{B}(\mathrm{G})$, and

$$
\|\mathrm{h}\|_{\mathrm{B}} \leq\left\|\tilde{\mathrm{f}}_{\omega}\right\|_{\Lambda}\left\|\mathrm{g}_{\omega}\right\|_{\mathrm{R}} \leq 16\|\tilde{\mathrm{f}}\|_{2^{2}} 2^{1 / 2}\left\|\mathrm{~g}_{\omega}\right\|_{2}=2^{9 / 2}\|\tilde{\tilde{\mathrm{f}}}\|_{2}\|\hat{\mathrm{g}}\|_{2}=2^{9 / 2}\|\hat{\mathrm{h}}\|_{1} .
$$

Finally we prove the main result of this paper.

THEOREM. $\mathrm{E} \subset \Gamma$ is a $\Lambda$-set if and only if $\mathrm{A}_{\mathrm{E}}(\mathrm{G})=\mathrm{B}_{\mathrm{E}}(\mathrm{G})$.

Proof. We observe first that $\left(\mathrm{L}_{\mathrm{E}}^{2}(\mathrm{G})\right)^{*}=\mathrm{L}_{\mathrm{E}}^{2}(\mathrm{G})$. Now suppose $\mathrm{E}$ is a $\Lambda$-set. Then, as we noted earlier, $L_{E}^{2}(G)=K_{E}(G)$. Take $g \in R_{E}(G)$. Then

$$
\begin{aligned}
\|g\|_{R} & \geq \sup _{f \in K_{E}(G)} \frac{|\langle f, g\rangle|}{\|f\|_{\Lambda}} \geq \sup _{f \in K_{E}(G)} \frac{|\langle f, g\rangle|}{C\left\|_{f}\right\|_{2}} \\
& =\frac{1}{C} \sup _{f \in L_{E}^{2}(G)} \frac{|\langle f, g\rangle|}{\|f\|_{2}}=\frac{1}{C}\|g\|_{\left(L_{E}^{2}(G)\right)^{*}}=\frac{1}{C}\|g\|_{2} .
\end{aligned}
$$

Hence $R_{E}(G)=L_{E}^{2}(G), K_{-E}(G)=L_{-E}^{2}(G)$, and therefore

$$
K_{-E}(G) \otimes_{L^{1}(G)} R_{E}(G)=L_{-E}^{2}(G) \bigotimes_{I_{1}\left(G_{1}\right)} L_{E}^{2}(G) .
$$


Using Proposition 2 and the fact that $L_{-E}^{2}(G) \bigotimes_{L^{1}(G)} L_{E}^{2}(G)$ is isometrically equal to $A_{E}(G)$, we see that $A_{E}(G)=B_{E}(G)$.

Now suppose $A_{E}(G)=B_{E}(G)$. Then $B_{E}(G)^{*}=A_{E}(G)^{*}$. Since $A_{E}(G)^{*}$ can be identified with $\ell^{\infty}(E)$, the relation $B_{E}(G)^{*}=A_{E}(G)^{*}$ implies the existence of a constant $C$ with the property that for each $\phi \in \ell^{\infty}(\mathrm{E})$ and each $\varepsilon>0$, there exists a $\nu \in \mathrm{B}(\mathrm{G})^{*}$ such that $\hat{\nu}(\gamma)=\phi(\gamma)$ for all $\gamma \in \mathrm{E}$ and such that $\|\nu\|_{\mathrm{B}^{*}} \leq \mathrm{C}\|\phi\|_{\infty}+\varepsilon$. Since $B(G)^{*}$ can be identified with $M(K)$, there exists a $T \in M(K)$ such that $\widehat{T f}=\hat{\nu} \hat{f}$ and $\left\|\mathrm{T}_{\nu}\right\|_{\mathrm{M}(\mathrm{K})} \leq \mathrm{C}\|\phi\|_{\infty}+\varepsilon$.

Now suppose $f \in L_{E}^{2}(G)$. By Proposition 3, there exists a \pm 1 -valued function $\phi$ on $E$ such that $\phi \hat{\mathrm{f}}$ is the Fourier transform of a function in $K_{E}(G)$. Since $\hat{\mathbf{f}}(\gamma)=0$ for all $\gamma \notin \mathrm{E}$, we see that

$$
\hat{\mathrm{f}}=\phi(\phi \hat{\mathrm{f}})=\hat{\nu}(\hat{\nu} \hat{\mathrm{f}})=\hat{\nu}(\widehat{\mathrm{Tf}})=(\mathrm{T}(\mathrm{Tf}))^{\wedge},
$$

and therefore $\mathrm{f}=\mathrm{T}(\mathrm{Tf})$. But $\mathrm{Tf} \in \mathrm{K}(\mathrm{G})$, by the choice of $\phi$; moreover, $\mathrm{T} \in \mathrm{M}(\mathrm{K})$; therefore $\mathrm{f} \epsilon \mathrm{K}_{\mathrm{E}}(\mathrm{G})$ and $\|\mathrm{f}\|_{\Lambda} \leq(\mathrm{C}+\varepsilon) 16\|\mathrm{f}\|_{2}$. This shows that $\mathrm{L}_{\mathrm{E}}^{2}(\mathrm{G})=\mathrm{K}_{\mathrm{E}}(\mathrm{G})$, and this is equivalent to the assertion that $\mathrm{E}$ is a $\Lambda$-set.

\section{REFERENCES}

1. G. Benke, Arithmetic structure and lacunary Fourier series. Proc. Amer. Math. Soc. 34 (1972), 128-132.

2. R. E. Edwards, E. Hewitt, and K. A. Ross, Lacunarity for compact groups. I. Indiana Univ. Math. J. 21 (1971/72), 787-806.

3. A. Figà-Talamanca, Multipliers of p-integrable functions. Bull. Amer. Math. Soc. 70 (1964), 666-669.

4. - Translation invariant operators in $\mathrm{L}^{\mathrm{P}}$. Duke Math. J. 32 (1965), 495501.

5. A. Figà-Talamanca and D. Rider, A theorem of Littlewood and lacunary series for compact groups. Pacific J. Math. 16 (1966), 505-514.

6. M.-P. Malliavin-Brameret and P. Malliavin, Caractérization arithmétique d'une classe d'ensembles de Helson. C. R. Acad. Sci. Paris Sér. A-B 264 (1967), A192-A193.

7. M. A. Rieffel, Induced Banach representations of Banach algebras and locally compact groups. J. Functional Analysis 1 (1967), 443-491.

8. - Multipliers and tensor products of $\mathrm{L}^{\mathrm{P}}$-spaces of locally compact groups. Studia Math. 33 (1969), 71-82.

9. W. Rudin, Fourier analysis on groups. Interscience Publ., New York, 1962.

10. A. Zygmund, Trigonometric series. Vol. I. Second edition. Cambridge Univ. Press, London-New York, 1968. 
